\title{
Cryoelectrolysis - electrolytic processes in a frozen physiological saline medium
}

\author{
Franco Lugnani $^{1}$, Matteo Macchioro ${ }^{2}$, Boris Rubinsky ${ }^{\text {Corresp. }} 3$ \\ 1 Clinica Santa Elena, Malaga, Spain \\ 2 Hippocrates D.O.O, Divaca, Slovenia \\ 3 Department of Bioengineering and Department of Mechanical Engineering, University of California, Berkeley, Berkley, California, United States \\ Corresponding Author: Boris Rubinsky \\ Email address: rubinsky@berkeley.edu
}

\section{Abstract}

Background: Cryoelectrolysis is a new minimally invasive tissue ablation surgical technique that combines the ablation techniques of electrolytic ablation with cryosurgery. The goal of this study is to examine the hypothesis that electrolysis can take place in a frozen aqueous saline solution.

Method: To examine the hypothesis we performed a cryoelectrolytic ablation protocol in which electrolysis and cryosurgery are delivered simultaneously in a tissue simulant made of physiological saline gel with a pH dye. We measured current flow, voltage and extents of freezing and pH dye staining.

Results: Using optical measurements and measurements of currents, we have shown that electrolysis can occur in frozen physiological saline, at high subzero freezing temperatures, above the eutectic temperature of the frozen salt solution. It was observed that electrolysis occurs when the tissue resides at high subzero temperatures during the freezing stage and essentially throughout the entire thawing stage. We also found that during thawing, the frozen lesion temperature raises rapidly to high subfreezing values and remains at those values throughout the thawing stage. Substantial electrolysis occurs during the thawing stage. Another interesting finding is that electro-osmotic flows affect the process of cryoelectrolysis at the anode and cathode, in different ways.

Discussion: The results showing that electrical current flow and electrolysis occur in frozen saline solutions imply a mechanism involving ionic movement in the fluid concentrated saline solution channels between ice crystals, at high subfreezing temperatures. Temperatures higher than the eutectic are required for the brine to be fluid. The particular pattern of temperature and electrical currents during the thawing stage of frozen tissue, can be explained by the large amounts of energy that must be removed at the outer edge of the frozen lesion because of the solid/liquid phase transformation on that interface.

Conclusion: Electrolysis can occur in a frozen domain at high subfreezing temperature, probably above the eutectic. It appears that the most effective period for delivering electrolytic currents in cryoelectrolysis is during the high subzero temperatures stage while freezing and immediately after cooling has stopped, throughout the thawing stage. 
1 Cryoelectrolysis - electrolytic processes in a frozen physiological saline 2 medium.

3

Franco Lugnani ${ }^{1}$, Matteo Macchioro ${ }^{2}$, Boris Rubinsky ${ }^{3}$

${ }^{1}$ Clinica Santa Elena. Malaga, Spain

${ }^{2}$ Hippocrates D.O.O. Divaĉa, Slovenia

${ }^{3}$ Department of Bioengineering and Department of Mechanical Engineering. University of California Berkeley. Berkeley, CA 94720 USA

Corresponding author:

Boris Rubinsky ${ }^{3}$

Email address: brubinsky@gmail.com

\begin{abstract}
:
Background: Cryoelectrolysis is a new minimally invasive tissue ablation surgical technique that combines the ablation techniques of electrolytic ablation with cryosurgery. The goal of this study is to examine the hypothesis that electrolysis can take place in a frozen aqueous saline solution. Method: To examine the hypothesis we performed a cryoelectrolytic ablation protocol in which electrolysis and cryosurgery are delivered simultaneously in a tissue simulant made of physiological saline gel with a pH dye. We measured current flow, voltage and extents of freezing and $\mathrm{pH}$ dye staining.
\end{abstract}

Results: Using optical measurements and measurements of currents, we have shown that electrolysis can occur in frozen physiological saline, at high subzero freezing temperatures, above the eutectic temperature of the frozen salt solution. It was observed that electrolysis occurs when the tissue resides at high subzero temperatures during the freezing stage and essentially throughout the entire thawing stage. We also found that during thawing, the frozen lesion temperature raises rapidly to high subfreezing values and remains at those values throughout the thawing stage. Substantial electrolysis occurs during the thawing stage. Another interesting finding is that electro-osmotic flows affect the process of cryoelectrolysis at the anode and cathode, in different ways.

Discussion: The results showing that electrical current flow and electrolysis occur in frozen saline solutions imply a mechanism involving ionic movement in the fluid concentrated saline solution channels between ice crystals, at high subfreezing temperatures. Temperatures higher than the eutectic are required for the brine to be fluid. The particular pattern of temperature and electrical currents during the thawing stage of frozen tissue, can be explained by the large amounts of energy that must be removed at the outer edge of the frozen lesion because of the solid/liquid phase transformation on that interface.

Conclusion: Electrolysis can occur in a frozen domain at high subfreezing temperature, probably above the eutectic. It appears that the most effective period for delivering electrolytic currents in cryoelectrolysis is during the high subzero temperatures stage while freezing and immediately after cooling has stopped, throughout the thawing stage. (331 words) 
44

45

46

47

48

49

50

51

52

53

54

55

56

57

58

59

60

61

62

63

64

65

66

67

68

69

70

71

72

73

74

75

76

77

78

79

80

81

82

83

84

85

86

\section{=Introduction.}

Tissue ablation with minimally invasive and non-invasive methods has emerged as an important branch of surgery. Various physical and chemical phenomena are used to ablate tissue, each with their advantages and disadvantages, and particular applications. For example, thermal ablation with nanoparticles (Kennedy, Bickford et al. 2011), thermal ablation with radiofrequency electromagnetic waves (Gazelle, Goldberg et al. 2000), thermal ablation with freezing, cryosurgery (Rubinsky 2000), chemical ablation that employs the products of electrolysis (Nilsson, von Euler et al. 2000) and non-thermal irreversible permeabilization of the cell membrane, non-thermal irreversible electroporation, (Rubinsky 2010). Recently, our group has become involved in studying combinations of these ablation techniques. The combinations examined include: electrolysis and electroporation; cryosurgery and electroporation; and cryosurgery and electrolysis (Lugnani, Zanconati et al. 2015, Rubinsky, Guenther et al. 2015, Stehling, Guenther et al. 2016). This paper pertains to the latter, the combination of cryosurgery and electrolysis, termed cryoelectrolysis (Lugnani, Zanconati et al. 2015); which is a largely unexplored process. Cryoelectrolysis, is marked by its potential to utilize the advantages of both cryosurgery and electrolytic ablation while overcoming their disadvantages. First, a brief review on the principles and attributes of cryosurgery and electrolytic ablation when used separately, followed by the principles of cryoelectrolysis and a description of the hypothesis examined in this work.

\section{Cryosurgery}

Cryosurgery is the ablation of undesirable tissues by freezing (Rubinsky 2000). The procedure employs a cryogenic fluid internally cooled cryosurgical probe, inserted in the undesirable tissue. The freezing propagates from the cryoprobe surface outward to freeze and, hopefully, thereby ablate the entire undesirable tissue. An important finding in cryosurgery is that the extent of freezing can be monitored in real time, by essentially every medical imaging techniques (Gilbert, Onik et al. 1984, Onik, Cooper et al. 1984, Rubinsky, Gilbert et al. 1993). This facilitates real time control over the extent of freezing. However, it was also found that cells can survive freezing at high subzero freezing temperatures. Therefore, cells can survive on the outer rim of the frozen lesion or around blood vessels, within the frozen lesion. Thus, the extent of freezing seen on medical imaging does not correspond to the extent of cell death. Currently, to increase the probability that all the cells in the frozen lesion are ablated, surgeons employ two to three cycles of freezing and thawing, which makes the procedure excessively long. Also, attempts are made to enhance cell death throughout the frozen lesion, by chemical means (Baust, Hollister et al. 1997, Koushafar, Pham et al. 1997, Clarke, Baust et al. 2001, Mir and Rubinsky 2002). A disadvantage of the chemical methods is the need to inject chemicals in the treated volume; a procedure that suffers from lack of control and precision.

Electrolytic Ablation. 
87 Electrolytic ablation, also known as Electro-Chemical Therapy (EChT), is a tissue ablation 88 technique that employs products of electrolysis for cell ablation (Nilsson, von Euler et al. 2000). 89 In EChT a direct electric current is delivered to the treatment field through electrodes that are 90 inserted in the treated tissue. New chemical species are generated at the interface of the 91 electrodes and tissue as a result of the electric potential driven transfer between the electrode 92 electrons and ions or atoms in the tissue. The various chemical species produced near the 93 electrodes diffuse away from the electrodes, into tissue, in a process driven by differences in 94 electrochemical potential. Tissue ablation by electrolysis is caused by two factors: the cytotoxic 95 environment developing due to local changes in $\mathrm{pH}$, as well as the presence of some of the new 96 chemical species formed during electrolysis. Electrolytic ablation requires very low direct 97 currents (tens to hundreds of $\mathrm{mA}$ ) and very low voltages (single to low tens of Volts) (Nilsson, von 98 Euler et al. 2000). This is advantageous, because it makes the devices used for this technology 99 extremely simple and safe. However, the procedure is long, from tens of minutes to hours. The

100

101

102

103

104

105

106

107

108

109

110

111

112

113

114

115

116

117

118

119

120

121

122

123

124

125

126

127

128

129 length is related to the slow diffusion of electrochemically produced species in tissue and the need for high concentrations of electrolytic products to cause cell death. A clinical study on tissue ablation with electrolysis states that- "Currently, a limitation of the technique is that it is time consuming" (Fosh, Finch et al. 2002, Fosh, Finch et al. 2003).

\section{Cryoelectrolysis}

The idea for tissue ablation by cryoelectrolysis, i.e. a combination of cryosurgery and electrolytic ablation, emerged from fundamental studies on the process of freezing in physiological saline solutions (Rubinsky 1983),(Rubinsky and Ikeda 1985, Rubinsky, Lee et al. 1987, Rubinsky and Pegg 1988, Rubinsky, Lee et al. 1990, Ishiguro and Rubinsky 1994). Figure 1 is a compendium of data from a number of our earlier studies and is brought here in a modified form, to facilitate a better understanding of the concept. Panels A, B, C and D, illustrate a series of events that occur on the solid-liquid interface during the solidification process in physiological saline. These events are driven by a thermodynamic condition known as constitutional supercooling (Rubinsky 1983). Constitutional supercooling predicts that even in a one dimensional solidification process, the solid/liquid change of phase interface is thermodynamically unstable and cannot remain planar. The sequence of panels, $A, B, C$, show how the interface becomes perturbed during the freezing process. Finger like ice crystals form and develop as dendritic structures. Ice has a very tight crystallographic structure and cannot contain any solutes. Therefore, the solutes previously contained in the volume now occupied by ice gather in the liquid between the ice crystal fingers. Panel $D$, shows the ultimate outcome of the freezing process in saline. High concentration brine solutions reside between finger like ice crystals. The concentration of the brine increases towards lower temperatures, until it reaches the eutectic at about $-21.1{ }^{\circ} \mathrm{C}$. Panels $\mathrm{E}, \mathrm{F}, \mathrm{G}$ show results from experiments in which we froze saline solutions with red blood cells. Panel $E$ is from the higher temperature tip of the finger like ice crystal structures. Panel $\mathrm{F}$ is for a lower temperature and panel $G$, is a further lower temperature. The white arrows point to the brine channels. It is evident that as the temperature decreases the volume of the channels decrease and concentration of brine increases. Panel $\mathrm{H}$ is a low temperature scanning electron micrograph of frozen liver. Here, ice forms inside the blood vessels (BV) and sinusoids (s) and the concentrated 
130 brine (light areas) surrounds the ice crystals and is in contact with the cells. The white arrow 131 points to the concentrated brine and cells.
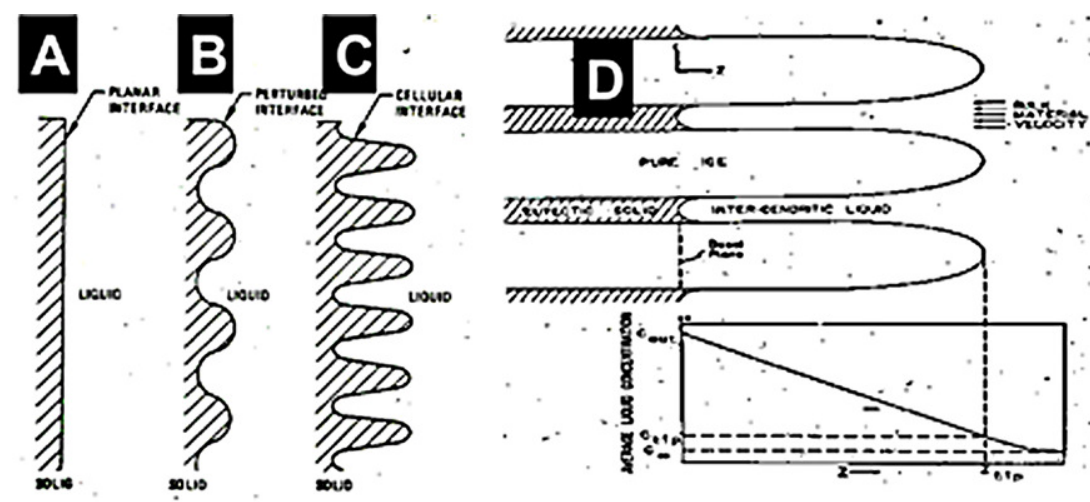

132

133

134

135

136

137

138

139

140

141

142

143

144

145

146

147

148

149

150

151

152

153

154

155
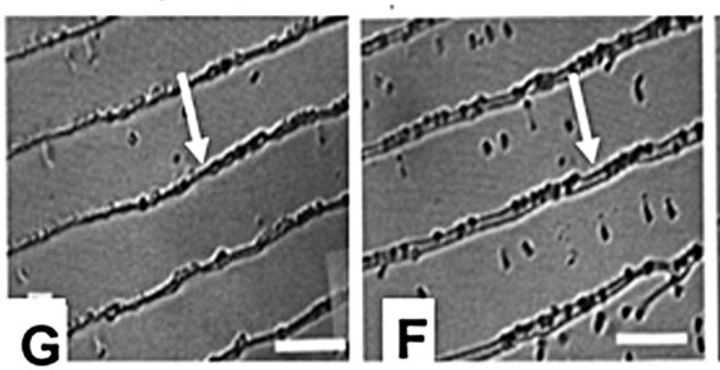

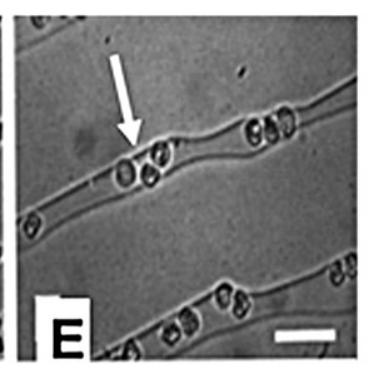

Figure 1

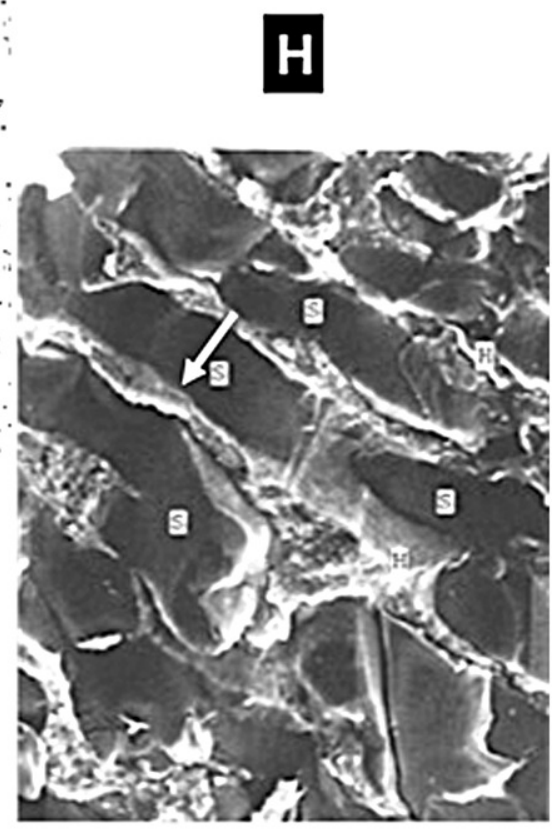

Cryoelectrolysis combines cryosurgery with electrolysis to overcome the limitations of cryosurgery and electrolysis used separately. The idea for the concept of cryoelectrolytic ablation was inspired by the findings described above, namely, that freezing of tissue increases the concentration of solutes around cells, by removing the water from the solution in the form of ice (Rubinsky and Pegg 1988). Freezing also causes cell membrane lipid phase transition, disrupts the cell membrane lipid bilayer and causes it to become permeabilized (Mir and Rubinsky 2002). From the data in Figure 1, it occurred to us that freezing of tissue in the presence of products of electrolysis will increase the concentration of the products of electrolysis around the cell. Furthermore, freezing induced cell membrane permeabilization will expose the interior of cells to the products of electrolysis and enhance cell death. The permeabilization of the cell membrane by freezing should decrease the concentration of the electrolytic products needed to cause cell death. Because the production of the electrolytic products is a time dependent reaction, decreasing the amount of electrolytic compounds needed for cell ablation, should shorten the time of an electrolytic induced mechanism of cell ablation. This is the basic principle of the cryoelectrolytic ablation concept proposed in (Lugnani, Zanconati et al. 2015). In that concept, the targeted tissue is first treated with electrolysis to generate products of electrolysis in the targeted volume; after which the targeted tissue is frozen to increase the local concentration and the exposure of the cell interior to the products of electrolysis in the frozen lesion. Theoretically the cryoelectrolysis combination should require lower concentrations of products of electrolysis i.e. shorter period of electrolysis and only one freeze thaw cycle. This should yield a shorter procedure than conventional electrolytic ablation or multiple freeze-thaw cycles of cryosurgery and, increase cell ablation in the frozen lesion by the dual mechanisms of 
156

157

158

159

160

161

162

163

164

165

166

167

168

169

170

171

172

173

174

175

176

177

178

179

180

181

182

183

184

185

186

187

188

189

190

191

192

193

194

195

196

197

198

199

freezing and electrolysis in the frozen lesion. The ability to image the extent of the frozen region, combines the advantages of real time image monitoring of cryosurgery with enhanced cell ablation by the combination freezing and electrolysis, in the frozen region.

Our first study on cryoelectrolysis was designed to examine the hypothesis that the combination of electrolysis and freezing, delivered as described above, is more effective at cell ablation than either electrolysis or freezing alone. The first study employed a protocol in which electrolysis was delivered first, followed by freezing. Experiments on animal tissue have confirmed our hypothesis and have shown that cryoelectrolysis is more effective at cell ablation than either cryosurgery or electrolytic ablation, alone (Lugnani, Zanconati et al. 2015).

While a protocol that employed first electrolysis and then freezing is faster than conventional electrolysis or the use of several freeze thaw cycles in conventional cryosurgery, the study in this paper was designed to explore an idea that may lead to a protocol that may be even faster. We think that the time of the procedure would be shorter, if, electrolysis and freezing, which are both diffusion limited processes, could be done simultaneously. The idea for this new concept was inspired by the same known, fundamental observation, described in regards to Fig.1; that freezing of tissue increases the concentration of solutes around cells, by removing the water from the solution in the form of ice (Rubinsky and Pegg 1988). These high concentration of solutes form brine channels within the frozen tissue (Rubinsky, Lee et al. 1987, Rubinsky, Lee et al. 1990, Ishiguro and Rubinsky 1994). The hypothesis that we have set to examine in this study is that the channels of high concentration brine in a frozen saline medium could serve as electrical conduits for the process of electrolysis. Therefore, while ice is not electrically conductive, electrolysis could be done through the high concentration brine channels in the frozen region, simultaneously with freezing and thawing.

\section{Materials and Methods}

The goal of this study is to examine the hypothesis that electrolysis can occur in frozen saline. In our study we employed a physiological saline gel to simulate tissue and used a modified commercial cryosurgery probe to deliver both cold and to serve as the electrolysis probe. The extent of freezing was monitored visually through change in opacity during freezing and the extent of the electrolysis was monitored also visually using a pH dye. Voltage and electrical current was measured throughout the experiments, to ascertain if and how electrical current flows through the frozen medium.

\section{Materials}

A physiological saline based agar was used to simulate tissue. One liter of water was mixed with 9 grams $\mathrm{NaCl}$ and 7 grams of agarose (UltraPure Agarose, Invitrogen). The solution was stirred and heated for 10 minutes and then removed from heat. Two $\mathrm{pH}$ indicator dyes were added after 
200 five minutes of cooling. For analysis of electrolysis near the anode, methyl red (Sigma-Aldrich ${ }^{\circledR}$,

201

202

203

204

205

206

207

208

209

210

211

212

213

214

215

216

217

218

219

220

221

222

223

224

225

226

227

228

229

230

231 St. Louis, MO, USA), $1 \mathrm{~mL}$ per $100 \mathrm{~mL}$ agar solution, was used. For analysis of electrolysis near the cathode we used Phenolphthalein Solution 0.5 wt. \% in Ethanol (Sigma-Aldrich) at a concentration of $5 \mathrm{ml}$ per liter agar (or $1 \mathrm{ml}$ per $100 \mathrm{ml}$ agar solution) solution. The agar was cast in a $20 \mathrm{~cm}$ diameter cylindrical glass vessel whose radial walls were coated with a $200 \mu \mathrm{m}$ thick copper foil. The height of the gel cast is $4 \mathrm{~cm}$.

\section{Experimental devices and set-up}

The two panels in Fig. 2, show photographs of the experimental setup. For the cryoelectrolysis experiment we used a Endocare ${ }^{\circledR}$ R2.4 cryoprobe with a diameter of $2.4 \mathrm{~mm}$ connected to an Endocare ${ }^{\circledR}$ single port control console device regulating flow duration and monitoring feed-back temperatures (Endocare Inc. Austin, TX, USA). The probe is supplied by a pressurized Argon gas container through the control console, at a constant pressure of 3000 psi. The cooling of the Endocare ${ }^{\circledR}$ stainless steel cryoprobe is through a Joule-Thomson internal valve. The cooling process is typical to all Endocare ${ }^{\circledR}$ cryoprobes of this type. The probe temperature reaches $180^{\circ} \mathrm{C}$, at a rate of cooling governed in part by the thermal environment in which the probe is inserted. A $30 \mu \mathrm{m}$ foil of gold was wrapped several times around the cryoprobe, to minimize the participation of the electrode metal in the process of electrolysis. The metal body of the probe was connected to a DC power supply (Agilent E3631A, Santa Clara CA, USA), to also serve as an electrolysis electrode. In a typical experiment the cryoelectrolysis probe was inserted vertical into the center of the gel. The electrical circuit consists of the power supply, the cryoelectrolysis probe electrode in the center of the gel, the gel and the copper electrode around the gel vessel. The gel was infused with methyl red when the cryoelectrolysis probe served as the anode and with phenolphthalein when the probe served as a cathode. A $1 \mathrm{~mm}$ T type thermocouple (Endocare $\AA$ ) was inserted to the vicinity of the cryoprobe at a distance of less than $5 \mathrm{~mm}$ from the outer surface of the probe, as shown in Fig. 2. The temperature was recorded continuously, throughout the experiment. It should be emphasized that this is not the temperature at the probe, but rather in the gel at a distance from the probe. A camera was focused on the experimental setup to continuously record the position of the change of phase interface, the position of the $\mathrm{pH}$ front, the voltage, current and time.

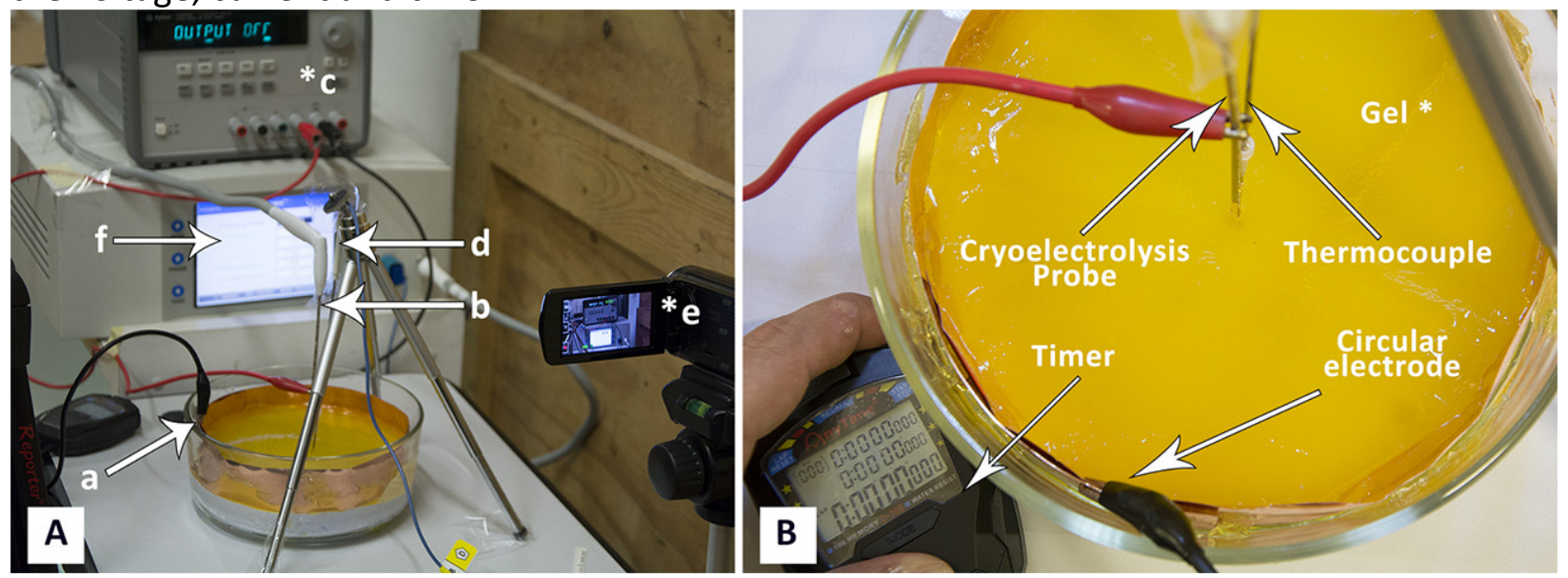

Figure 2 
232

233

234

235

236

237

238

239

240

241

242

243

244

245

246

247

248

249

250

251

252

253

254

255

256

257

258

259

260

261

262

263

264

265

266

267

268

269

270

271

272

273

274

275

\section{Experimental protocol}

In this study, we performed first a number of experiments with electrolysis only, without freezing, to determine the currents and time of application needed to obtain measureable data for the extent of electrolysis in our experimental set-up. From these experiments we chose values of 400 $\mathrm{mA}, 200 \mathrm{~mA}$ and $50 \mathrm{~mA}$. The range of electrical currents tested are typical to clinical electrolytic ablation procedures, (Nilsson, von Euler et al. 2000, Lugnani, Zanconati et al. 2015). Similarly, preliminary experiments were performed with freezing only to evaluate the time of freezing needed to obtained measurable frozen lesions in our experimental configuration. From these results we chose ten minutes of freezing and fifteen minutes for thawing.

The following experimental procedure was employed in all the experiments. The experimental protocol was designed to examine all aspects of the hypothesis of this study; electrolysis before freezing, electrolysis during freezing and electrolysis during thawing. The electrical circuit, comprised of the cryoelectrolysis probe, the gel and the copper vessel walls, was connected to the power supply first. It remained connected throughout the experiment, during freezing and thawing. The first minute was electrolysis alone. The flow of cryogen began one minute after the circuit was connected to the power supply, initiating the freezing. Constant pressure of $3000 \mathrm{psi}$ was used to generate the Argon gas flow in a manner typical to clinical cryosurgical treatment with the cryosurgery probe we used. The flow of cryogen was delivered for ten minutes, during which the gel froze. This is the stage in which freezing and electrolysis were delivered simultaneously. After ten minutes, the flow of the cryogen was stopped and the frozen lesion was left to thaw, in situ. The electrical circuit remained connected to the power supply for additional 15 minutes after the flow of the cryogen was stopped. This represents the stage in which thawing and electrolysis occurs simultaneously.

We performed three repeats of each experiment with 400mA, $200 \mathrm{~mA}$ and $50 \mathrm{~mA}$ currents for both the central electrode anode and the central electrode cathode for a total of 18 cryoelectrolysis experiments with the protocol described above. The voltage was allowed to change to provide the desired current. However, the saturation voltage of the power supply used in this study is $25 \mathrm{~V}$ and the system cannot provide a higher voltage. Therefore, when changes in resistance demanded a voltage higher than $25 \mathrm{~V}$, the current dropped and eventually stopped.

\section{Results and discussion}

The primary goal of this study is to examine the hypothesis that electrolysis can occur in a frozen aqueous saline solution. We will bring here results that support the hypothesis.

Figure 3, presents a compilation of photographs that illustrate several important observations, typical to all the experiments performed in this study. Panels $3 \mathrm{~A}$, and 3B, are images of the progression of the $\mathrm{pH}$ front during a preliminary study in which there was only electrolysis, without freezing. The goal of these two panels is to illustrate the appearance of a typical process 
276 of electrolysis in a $\mathrm{pH}$ stained gel. The cryoelectrolysis probe served as the anode and delivered $277400 \mathrm{~mA}$. Panel 3A, shows the radially symmetric $\mathrm{pH}$ front around the anode. The panels show a 278 cylindrical $\mathrm{pH}$ stained region around the probe. This is the region in which the products of 279 electrolysis reside. The interface between the stained and unstained regions is referred in this 280 paper as the, $\mathrm{pH}$ front. The process of electrolysis was continued for several minutes and panel

281

282

283

284

285

286

287

288

289

290

291

292

293

294

295

296

297

298

299

300

301

302

303

304

305

306

307

308

309

310

311

312

313

314

315

316

317

318

319 3B shows the extent of electrolysis at a later time. Obviously the $\mathrm{pH}$ front has advanced, while remaining radially symmetric. The white arrow points to an observation of importance to cryoelectrolysis. Diffusion and iontophoresis driven electro-osmosis, are the physical mechanisms that cause the propagation of the $\mathrm{pH}$ front from the electrode outward. The electroosmotic flow is an important aspect of electrolytic ablation in tissue (Lugnani, Zanconati et al. 2015, Phillips, Raju et al. 2015, Phillips, Rubinsky et al. 2015, Rubinsky, Guenther et al. 2015, Rubinsky, Guenther et al. 2016). The flow is from the anode to the cathode. The white arrow points to a dark gap that has formed between the electrode and the gel. (Inserts in Fig 3 are magnified views of the region near the electrode) The gap was caused by the electro-osmotic driven flow of solution, away from the anode, towards the cathode. The later panels in this figure will illustrate the significance of this electro-osmotic flow to cryoelectrolysis.

Panels 3C, and 3D, are images of the progression of the $\mathrm{pH}$ stained region and of the frozen region during a typical cryoelectrolytic protocol of the type described in the materials and methods section. The cryoelectrolysis probe served as anode and delivered $400 \mathrm{~mA}$. Panel $3 \mathrm{C}$ shows the appearance of the frozen lesion at the end of the freezing stage of the protocol. The dashed arrow point to the edge of the frozen lesion. Panel 3D is a photograph from the same experiment taken several minutes after the cooling was stopped, while the power supply continued to deliver current to the electrical circuit. Two interesting observations emerge. While the extent of the frozen lesion in panel 3D has not changed from that in panel $3 \mathrm{C}$; the $\mathrm{pH}$ stained region has expanded beyond the frozen lesion. This demonstrates that the process of electrolysis can occur through ice, during the thawing stage. Similar observations were made with all the currents tested and in all the repeats. This is an important observation, which will be discussed later in the context of Figures 4 and 5. The white arrow shows that the electro-osmotic flow generated gap formed between the electrode and the gel during conventional electrolysis, also occurs during cryoelectrolysis. This further strengthens the evidence that electrolysis occurs through a frozen region.

Panels $3 \mathrm{E}$, and $3 \mathrm{~F}$, are images of the progression of the $\mathrm{pH}$ front (the $\mathrm{pH}$ stained area) and of the ice front (the frozen lesion) during a typical cryoelectrolytic protocol of the type described in the materials and methods section when the cryoelectrolysis probe served as the cathode and delivered $50 \mathrm{~mA}$. Obviously, the appearance of the treated areas in panels $3 \mathrm{E}$ and $3 \mathrm{~F}$ is completely different from that in panels $3 \mathrm{C}$ and $3 \mathrm{D}$. Panel $3 \mathrm{E}$ is from an earlier stage of the cryoelectrolysis protocol, during which, both electrical current and cryogen cooling, were delivered by the cryoelectrolysis probe, simultaneously. It is important to observe that both, a $\mathrm{pH}$ stained region and a frozen lesion have formed and they propagate away from the probe. However, in the case of a cathode centered electrode, the propagation is in an asymmetric way. The lack of symmetry is evident in comparison with panel 3C. The difference is caused by the direction of the electroosmotic flow, which in this case, is towards the cryoelectrolysis cathode probe. This generates a 
320 high flow rate of solution, at the cryoelectrolysis cathode probe - gel interface. We have observed 321 a flow of water gushing out at the interface between the cryoelectrolysis probe and the gel, 322 regardless of the current magnitude used and in all the cryoelectrolysis cathode probe study 323 repeats. The water also contains a mixture of gas (hydrogen from the reduction reaction near the 324 cathode). Evidence of the process can be seen from the red dots spread over the right hand side 325 of the gel (dotted arrow in panel 1EG). The red dots are caused by the splashed droplets of high $326 \mathrm{pH}$ fluid. The electro-osmotic pressure has caused various random and detrimental effects, when 327 the cryoelectrolysis probe is the cathode. For higher currents, of $200 \mathrm{~mA}$ and $400 \mathrm{~mA}$, the electro328 osmotic pressure driven flow has caused fractures and cracks in the gel. For the lower currents

329

330 331

332

333

334

335

336

337

338

339

340

341

342

343

344

345

346

347

348

349

350

351

352

353

354

355

356

357 of $50 \mathrm{~mA}$ it produced the lack of symmetry seen in panels 3E and 3F. The electro-osmotic pressure caused events, occur at random and the cracks formation is not predictable.

Panel 3E was taken during the last stage of the experiment; a stage in the typical cryoelectrolysis protocol in which the cooling was stopped and only electrolysis occurs through the frozen region that is thawing. This is at a similar stage in the protocol to that in which panel 3D photograph was taken. Here, we observe that the $\mathrm{pH}$ front has propagated irregularly both within and beyond the frozen lesion. The propagation of the $\mathrm{pH}$ front occurred while the frozen lesion still exists. This demonstrates that the process of electrolysis can occur through a frozen domain when the cryoelectrolysis probe is either anode or cathode. The lack of symmetry in the appearance of the $\mathrm{pH}$ front in panel 3F can be, probably, attributed to cracks that form in the gel because of the electro-osmotic pressure. These cracks favor certain directions of propagation of the electrolytic products flow. The magnified insert of the region near the cryoelectrolysis cathode probe provides further evidence on the effect of the electro-osmotic flow. The dark gap between the cryoelectrolysis anode probe and the gel in panels $3 C$ and $3 D$ does not form when the cryoelectrolysis probe is the cathode. In fact, the white arrows point to a bulging volume of ice formed in the vicinity of the cryoelectrolysis probe. The insert also shows a crack in the gel, filed with ice. While qualitatively similar results were observed in all the repeats of the cathode centered experiments, the quantitative appearance was different from repeat to repeat because of the random appearance of the electro-osmotic flow generated cracks.

In summary, this part of the study reveals two important physical phenomena related to cryoelectrolysis: a) electrolysis can occur through a frozen milieu at both, the anode and the cathode, b) electro-osmotic flows play an important part in the physical events that occur during cryoelectrolysis. Because of electro-osmotic flows the outcome of the procedure, is different between a cryoelectrolysis cathode probe and a cryoelectrolysis anode probe. The results tentatively suggest that it may be beneficial to use for cryoelectrolysis only the anode and employ a surface electrode (similar to that used in radiofrequency ablation) as the cathode. 


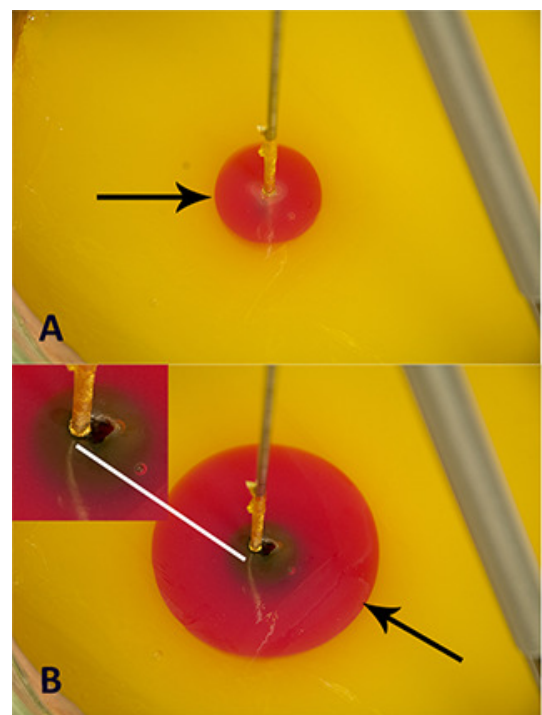

358

359

360

361

362

363

364

365

366

367

368

369
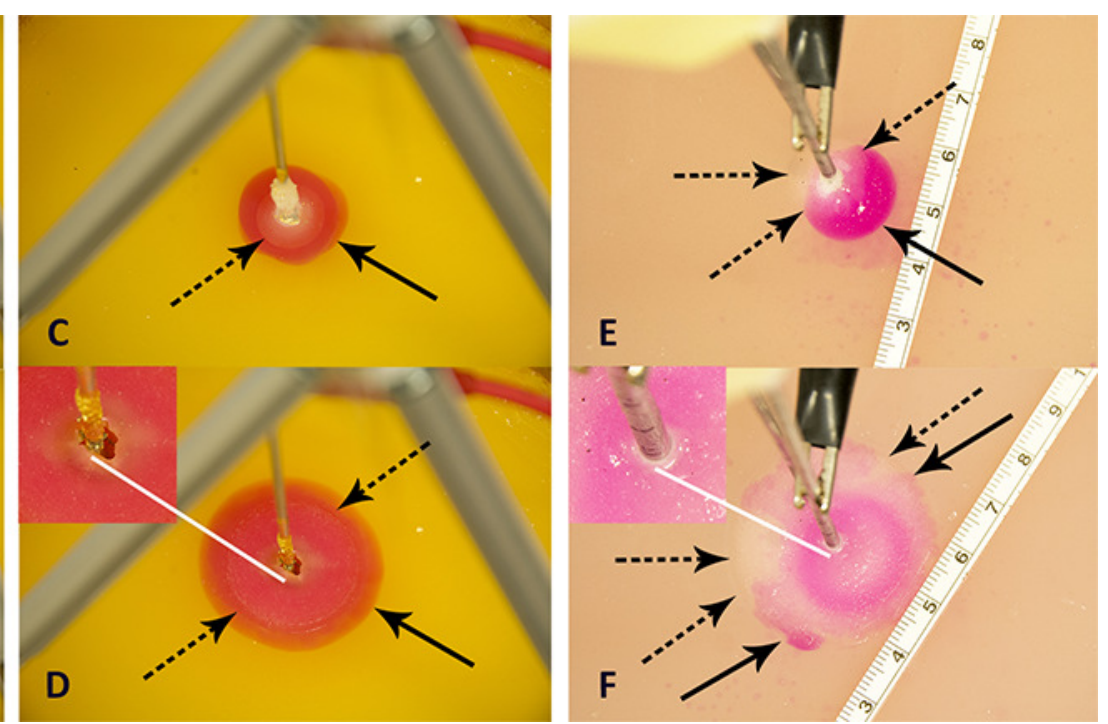

Figure 3

Figures 4 and 5 are typical to all the anode center experiments of this study. They were chosen to illustrate the events that are relevant to the hypothesis and which occur during a typical processes of cryoelectrolysis. We focus here on the anode center experiments because for this configuration, the results in the different repeats and with the different currents were similar, unlike for the cathode centered experiments. The cathode center experiments were different from experiment to experiment because of the random formation of electro-osmotic flow induced cracks. We will illustrate the observations with results in which the cryoelectrolysis probe was the anode and the current was set to, $200 \mathrm{~mA}$. 


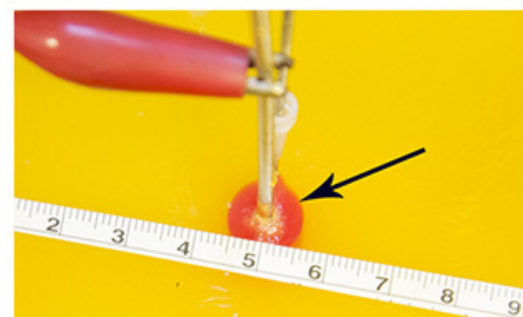

A
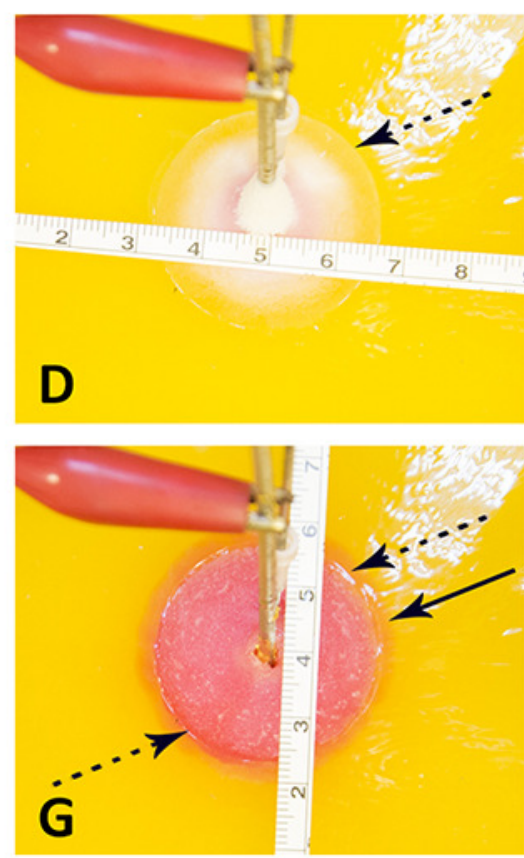

370

371

372

373

374

375

376

377

378

379

380

381

382

383

384

385
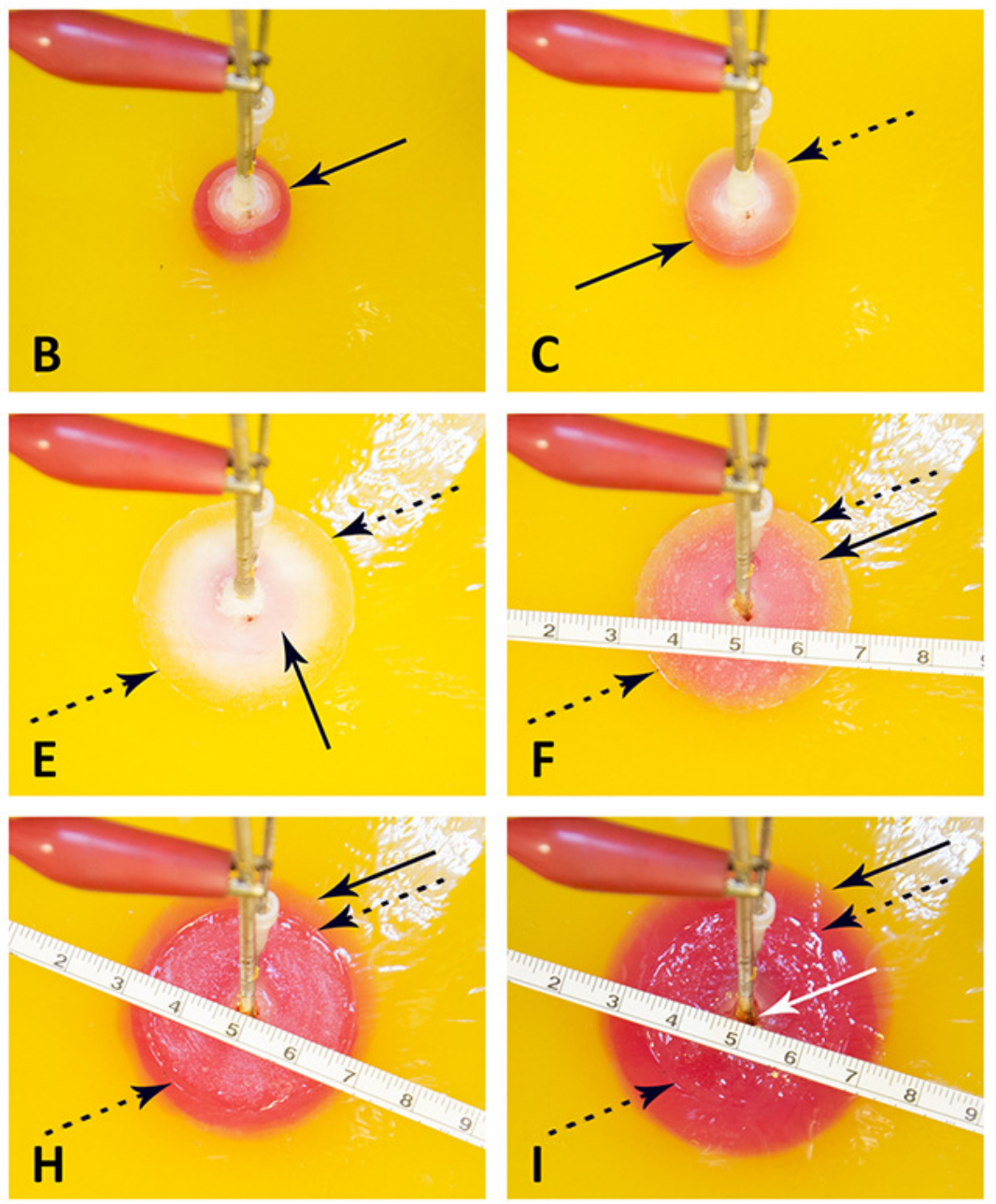

Figure 4

Figure 4 is a sequence of images showing the $\mathrm{pH}$ front and the ice front at different instances in time during the cryoelectrolysis protocol. Panel 4A, shows the appearance of the $\mathrm{pH}$ stained region, one minute after the start of the experiment, just prior to the start of the cooling process. Panel 4B shows the appearance of the ice front and of the $\mathrm{pH}$ front one minute after the start of freezing and two minutes after the start of the experiment. It is evident from comparison with panel $4 \mathrm{~A}$ that during this one minute of freezing, the ice front and the $\mathrm{pH}$ front have both advanced. This is an important observation as it demonstrates that electrolysis occurs during freezing. However, Panels 4C, and 4D show that after one minute of freezing, the pH front stops advancing (no electrolysis) while the ice front propagates further. This shows that there are conditions in which electrolysis does not occur in a frozen solution. Panels 4D to 4I, show that after the coolant has stopped flowing through the cryoprobe, the extent of the frozen lesion remains unchanged for a long period of time. However, the extent of the $\mathrm{pH}$ dye stained region increases in time and eventually extends beyond the frozen lesion. 


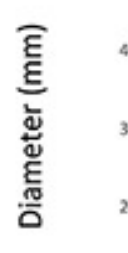

10

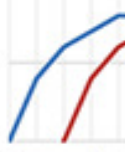
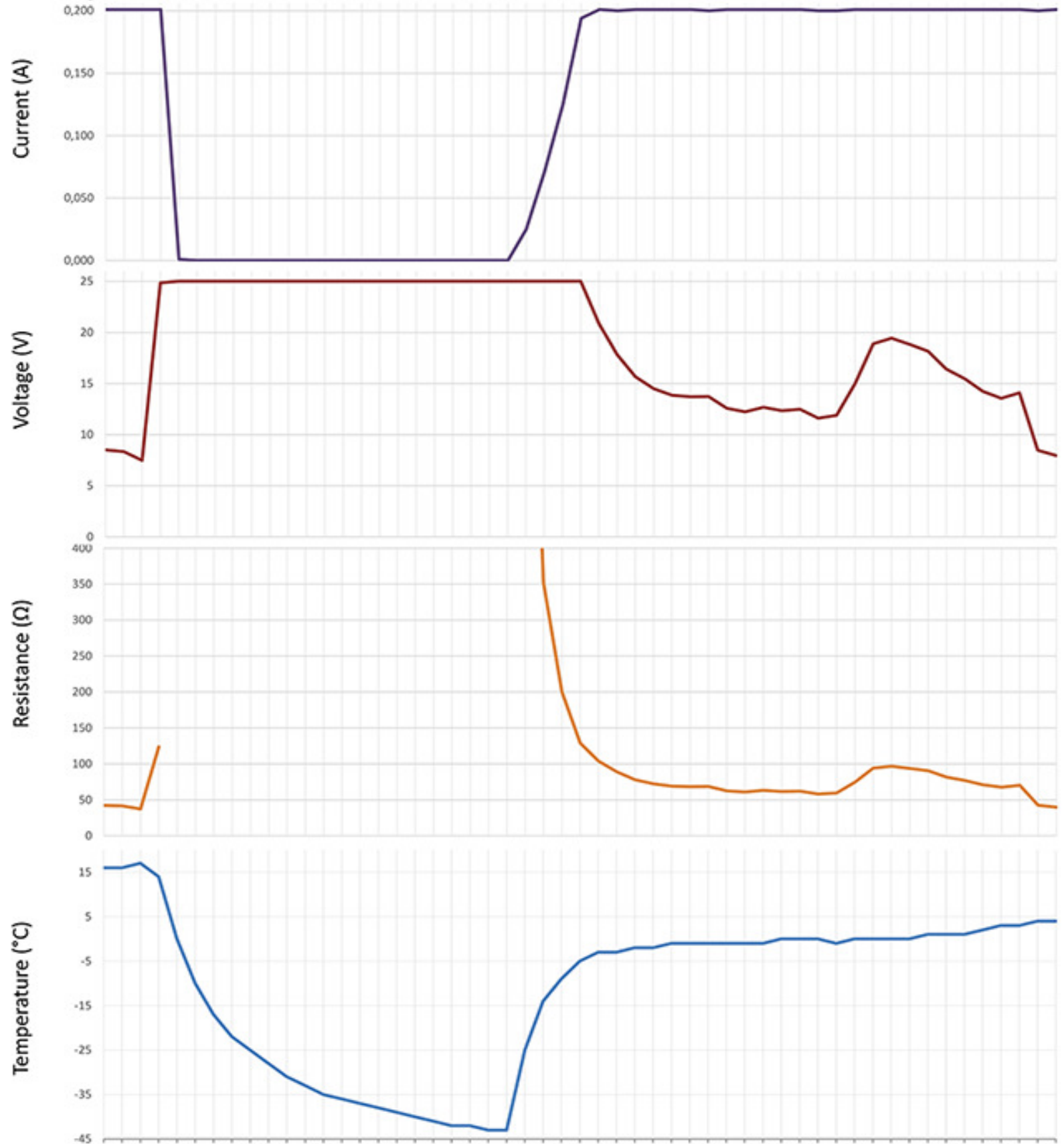

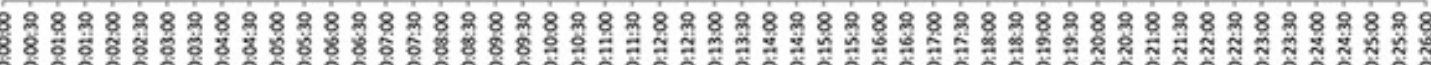

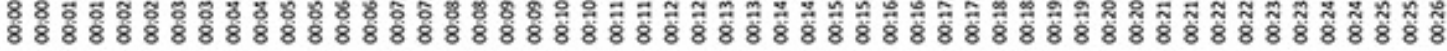

Figure 5 
387 Figure 5 is from the same experiment as Figure 4. It displays, the data measured during that

388

389

390

391

392

393

394

395

396

397

398

399

400

401

402

403

404

405

406

407

408

409

410

411

412

413

414

415

416

417

418

419

420

421

422

423

424

425

426

427

428

429

430 experiment. The panels show, from top to bottom: the diameters of the $\mathrm{pH}$ stained region and of the frozen lesion, the measured current, the measured voltage, the calculated resistance and the temperature of the thermocouple, as a function of time during the cryoelectrolysis process examined in this study. The first minute of the protocol is electrolysis, without freezing. Figure 5 shows that during this first minute the current is constant at $200 \mathrm{~mA}$, the temperature is constant at $15 \mathrm{C}$, the voltage is about $8 \mathrm{~V}$, resistance is constant and the extent of the $\mathrm{pH}$ dye stained region increases in time. All these are evidence of a process of electrolysis. Cooling the probe, began one minute after the start of the experiment. As soon as cooling began, the temperature measured by the thermocouple began to drop. (It should be emphasized that the thermocouple is at a distance from the probe, and does not measure the temperature of the probe, which is lower than the thermocouple measurement.) The other curves in Fig. 5 show that the diameter of the freezing zone increases in time, throughout the ten minutes of cooling. During the first minute of cooling (freezing) there is current and the extent of the $\mathrm{pH}$ dye stained region increases. The resistance increases, the voltage increases to the maximum that the power supply can deliver (25V) and the current decreases to zero after about one minute of freezing. The resistance becomes, in fact, infinite after one minute of freezing. Nevertheless, it is important to notice that during the first minute of freezing there is electrolysis and current flows through the frozen lesion. Cooling continues for ten minutes, during which the thermocouple measured temperature drops further, the frozen zone expands and no current flows through the frozen lesion. After ten minutes of cooling, the flow of the cryogen is stopped, while the power supply for electrolysis remains on. The thermocouple reading shows that the temperature in the frozen region begins to increase as soon as the cooling has stopped. However, an interesting phenomenon occurs. The temperature remains at a high subzero value, below the freezing temperature for the remainder of the experiment, i.e. the temperature around the probe (electrode) is below freezing. Visual observation displayed on the top panel in Fig. 5 and in Fig. 4 (panels $D$ to I) show that the extent of the frozen lesion does not change to the end of the experiment. Within a minute after the cooling has stopped and the temperature began to increase, the current increases, the voltage drops and the resistance drops. The $\mathrm{pH}$ dye stained region begins to increase and eventually becomes larger than the frozen lesion. Taken together this presents evidence that electrolysis occurs in the frozen lesion after freezing has stop.

The results displayed in Figs. 4 and 5 are typical to all the anode center experiments. They demonstrate that electrolysis occurs in a frozen saline solution at high subzero temperatures. The results are consistent with the hypothesis and are explained in the formulation of the hypothesis in the introduction and in Fig. 1. The mechanism responsible for electrolysis in a high subzero frozen media is associated with the process of freezing in solutions and tissues, as described in the introduction. Ice has a tight crystallographic structure and cannot contain any solutes. Constitutional supercooling dictates that during freezing of a solution, finger like ice crystals form and the salt is rejected along the ice crystals (Rubinsky 1983). High concentration salt solutions form along the ice crystals. This phenomenon occurs during freezing of any aqueous medium, in solutions (Ishiguro and Rubinsky 1994), gels (Preciado, Shandakumaran et al. 2003) and tissues (Rubinsky and Pegg 1988). While the electrical conductivity of ice is essentially zero, 
431 electrical currents can flow through these high concentration brine channels until the 432 temperature reaches the eutectic $-21.1^{\circ} \mathrm{C}$. Panels $1 \mathrm{E}, 1 \mathrm{~F}$ and $1 \mathrm{G}$, show that as the temperature 433 decreases, the channels become narrower, until eutectic is reached. Eutectic is a solid phase and 434 ionic movement ceases. This explain the observed increase in resistance during the first minute 435 of freezing and the decrease in resistance after cooling has stopped and the temperature of the 436 frozen tissue began to increase. This result is important in designing cryoelectrolysis protocols, 437 because it shows that electrolysis can occur in a frozen domain, only at high subzero 438 temperatures; most likely below the eutectic. Therefore, in cryoelectrolytic ablation, it should be 439 beneficial to reside longer at high subfreezing temperatures during the freezing stage. This is in 440 marked contrast to current cryosurgery freezing protocols in which freezing is done rapidly to 441 low subzero freezing temperatures.

442

443 The phenomena observed after cooling has stopped are particularly interesting and of value to 444 designing a cryoelectrolysis ablation protocol. Figure 5 shows that the temperature measured by 445 the thermocouple begins to raise as soon as the cooling stops. However, the measured temperature remains close to, albeit lower, than the phase transformation temperature for most of the remainder of the cryoelectrolysis protocol. This is a phenomenon we have observed and studied in the past (Rubinsky and Cravalho 1979, Hong and Rubinsky 1995). To better understand the phenomenon, we bring here Fig 6 . It is a qualitative depiction of results from mathematical analysis of thawing of frozen cylinders in (Rubinsky and Cravalho 1979, Hong and Rubinsky 1995). The figure shows that when a frozen domain begins to thaw from the exterior, as is also the case in the cryoelectrolysis protocol, the temperature of the frozen region raises rapidly towards the change of phase temperature. However, the melting, which propagates from the exterior of the frozen domain towards the interior is very slow, relative to the raise of the temperature in the frozen domain. Therefore, the frozen domain, stays at high subfreezing temperatures throughout the process of melting. 


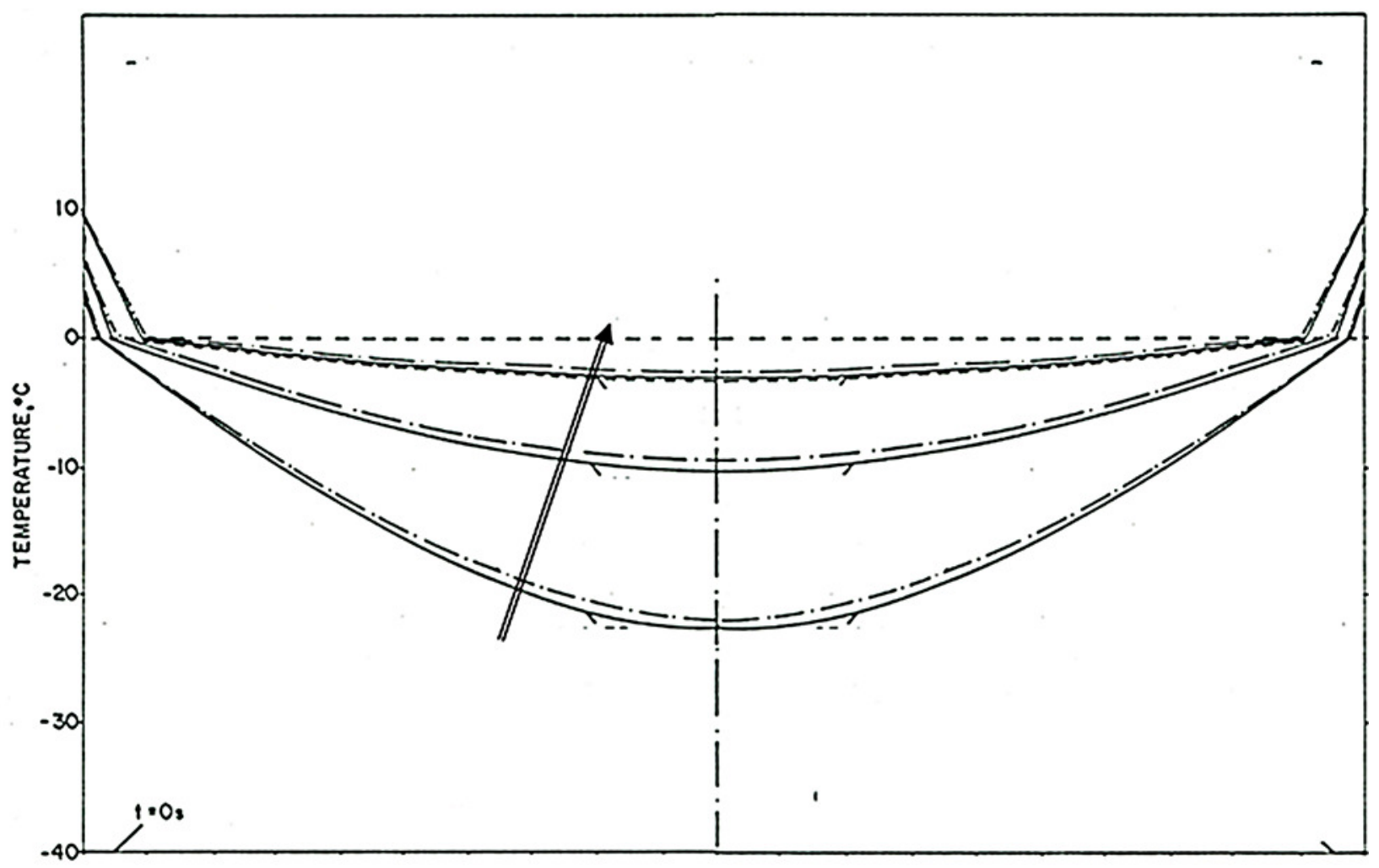

457

458

459

460

461

462

463

464

465

466

467

468

469

470

471

472

473

474

475

476

477

478

479

480

Figure 6

An explanation for this phenomenon was provided first in (Rubinsky and Cravalho 1979). The phenomenon is related to the fact that the change in enthalpy during phase transition of ice into water is very large relative to the change in enthalpy due to change in the temperature of the ice. Briefly, during melting, heat is extracted from the frozen domain, through the change of phase interface, by the environment surrounding the interface. The temperature of the change of phase interface is fixed by equilibrium thermodynamics of a two phase system at constant pressure. For physiological saline it is $-0.56{ }^{\circ} \mathrm{C}$. As long as there is an ice and water mixture in a domain, the temperature of that domain cannot exceed the thermodynamic phase transition temperature of the solution. The phase transformation process (melting) occurs only on the change of phase interface, which propagates very slowly, because the large change in enthalpy involved. Since the enthalpy associated with changes of temperature in the frozen domain are very small relative to the change in enthalpy by phase transformation, the temperature of the frozen region becomes elevated and reaches the phase transition temperature fast, throughout the frozen region; while the region is still frozen (Rubinsky and Cravalho 1979). Consequently, while the extent of the frozen regions remains essentially unchanged the end of cooling (panels $4 \mathrm{E}$ to $4 \mathrm{I}$ ) the temperature of the frozen region raises to become close and below the change of phase temperature, for a long period of time; Fig 5, bottom temperature curve. The temperature measurements in Figure 5 validate this explanation. The increase in the temperature of the frozen region has several effects. Figure 5 shows that there is a gradual increase in current and a decrease in resistance, soon after cooling stops. Consequently, there is a process of electrolysis, and the $\mathrm{pH}$ front expands beyond the margin of the frozen region, while the region is still frozen (panels 4E to $4 \mathrm{I}$ ). 
481

482

483

484

485

486

487

488

489

490

491

492

493

494

495

496

497

498

499

500

501

502

503

504

505

506

507

508

509

510

511

512

513

514

515

Figure 5 shows that indeed current begins to flow through the high subzero temperature region of frozen gel, soon after cooling stops. Unavoidable, flow of ionic current is associated with electrolysis and this is why the $\mathrm{pH}$ front advances while the tissue is still frozen, albeit at high subzero temperatures. The flow of current through the brine channels most likely elevated the local temperature of these channels and may cause local melting and expansion or collapse of the brine channels. It is possible that this phenomenon is responsible for the jumps in voltage measured occasionally (see Fig. 5). We have seen various sudden jumps in voltage, during the period after the cooling has stopped, in all the experiments.

The physiological effects of electrolysis during the thawing process remain to be examined with living tissue. However, we anticipate that the electrolysis during the thawing process will be effective at tissue ablation. While the phenomenon of concentrating the products of electrolysis by freezing, does not occur anymore, the cell membrane is still permeabilized by cold and provides access to the products of electrolysis. Furthermore, the thawing stage during cryosurgery is unavoidable long. Delivering current during that stage may have a dual effect. It may shorten the length of thawing because of the Joule heating effect and enhance cell death by the products of electrolysis. This is why delivering electrolytic currents during the thawing stage of cryoelectrolysis may be desirable.

\section{Conclusion}

The primary goal of this study was to examine the hypothesis that electrolysis can occur in frozen aqueous saline. The combined effect of freezing and electrolysis was studied in a tissue simulant made of a physiological solution of agar with $\mathrm{pH}$ dyes. The most important finding of this study is that electrolysis can occur in a frozen aqueous saline and the hypothesis is proven. To the best of our knowledge, this is the first time that electrolysis through ice was observed and reported. This finding is valuable for designing cryoelectrolysis protocols. It demonstrates that the processes of freezing and of electrolysis can be done simultaneously. It appears that the most effective period for delivering electrolytic currents is during the high subzero temperatures while freezing and immediately after cooling has stopped, throughout the thawing stage.

Acknowledgement: We are grateful to Dr. Liel Rubinsky who did the first work on cryoelectrolysis and whose work planted the seeds for this paper and to Mr. Paul Mikus for useful advice. 


\section{List of Figures:}

517

518
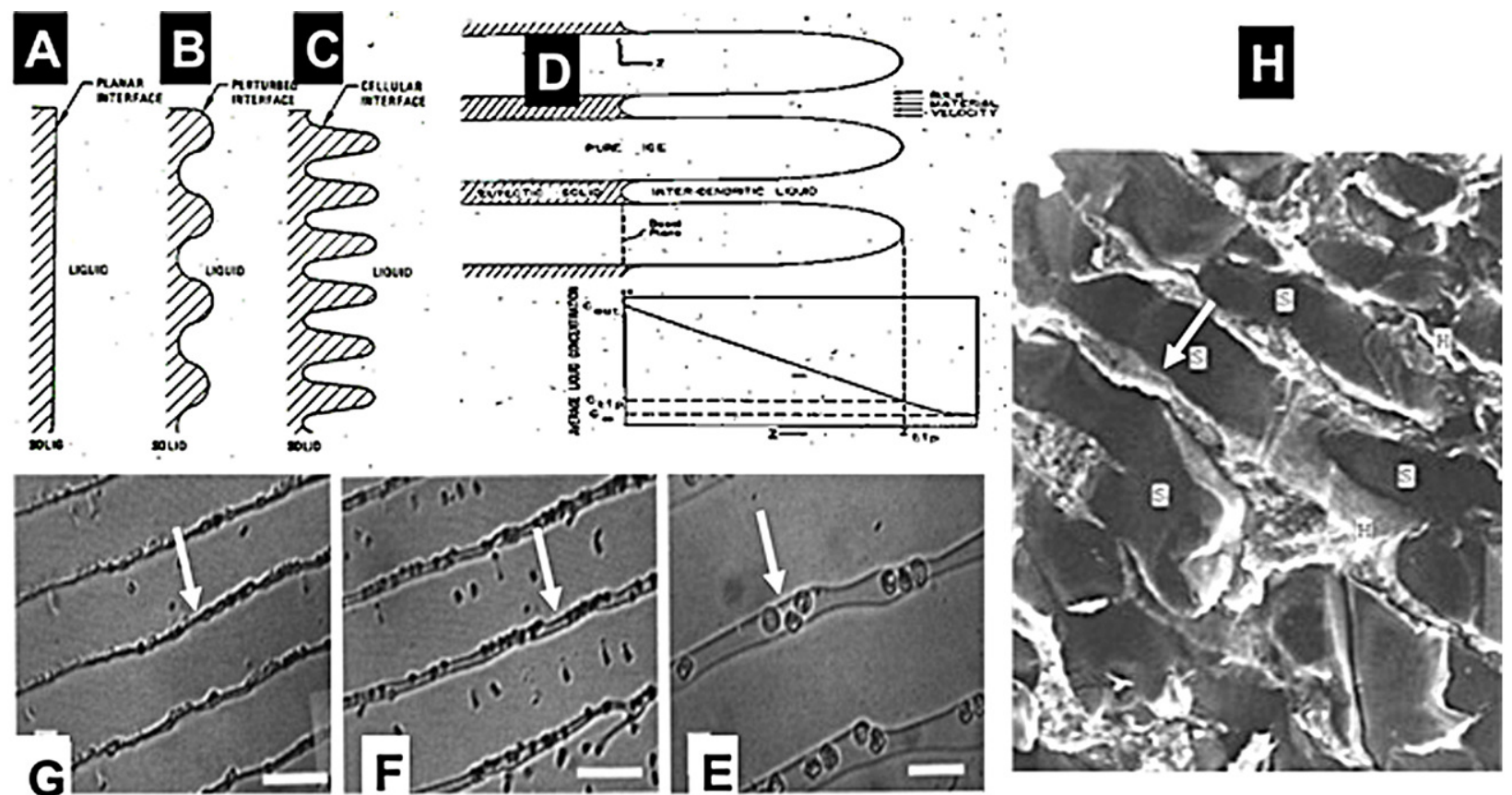

519

520

521

Figure 1: Compendium of schematic and experimental results to serve as an explanation for the

522 fundamental concepts of cryoelectrolysis. (This figure is a compendium of unpublished data

523 from one of the authors BR)

524

525 

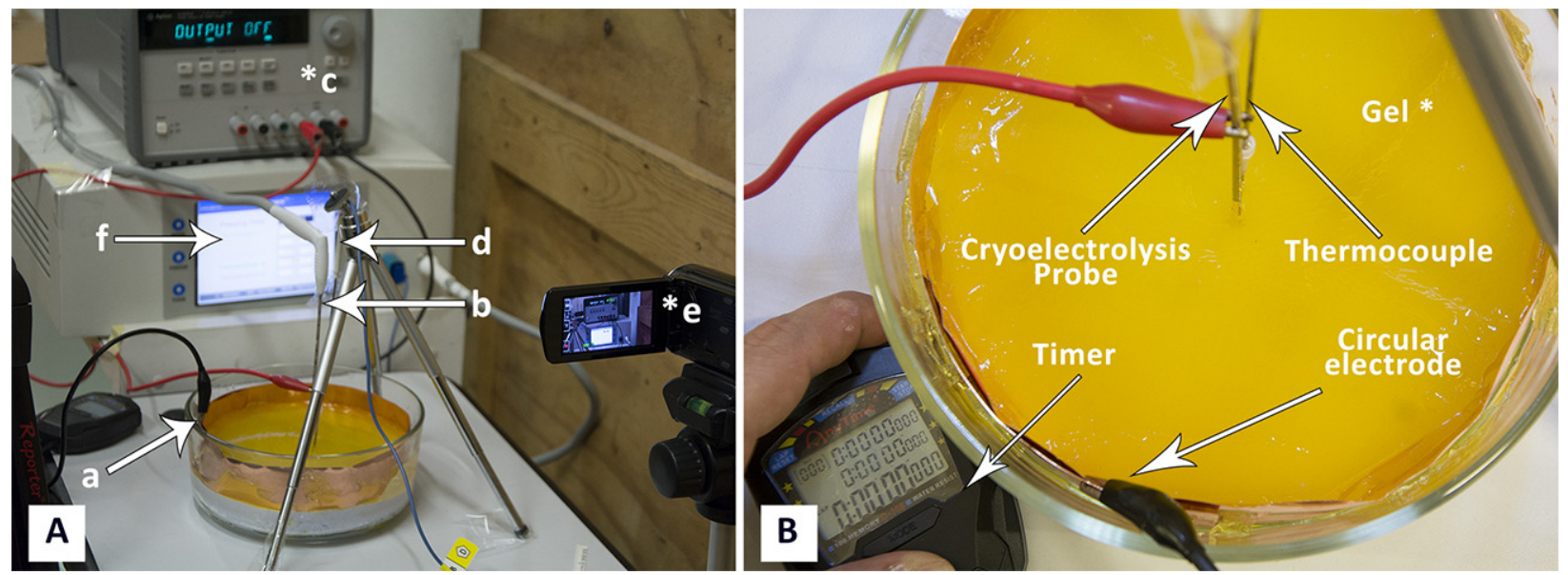

526

527

528

Figure 2: A) Photograph of experimental system: a - electrode on container surface, b-

529 cryoelectrolysis probe, $c-D C$ power supply, $d$ - thermocouple, $e$ - camera, $f$ - cryosurgery

530 probe pressure monitor; B) close-up of the gel and electrodes;

532

533

534 

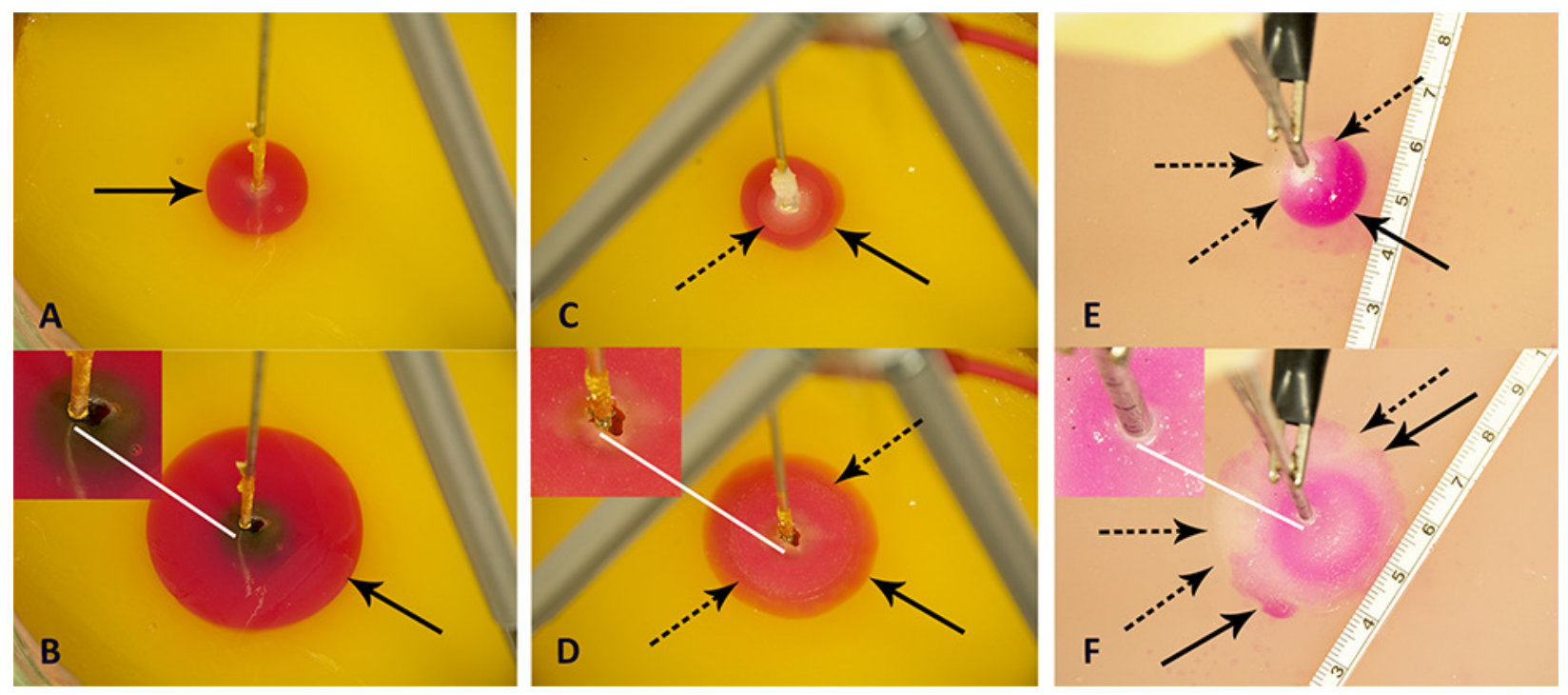

535

536

537

538

539

540

541

542

543

544

545

546

547

548

549

Figure 3: Illustration of typical cryoelectrolysis process. Photographs of the $\mathrm{pH}$ front and freezing front in different experiments: A) electrolysis only, $400 \mathrm{~mA}$ current, B) electrolysis only, $400 \mathrm{~mA}$ current at a later time from panel $A, C$ ) cryoelectrolysis with cryoelectrolysis probe as the anode, $400 \mathrm{~mA}, \mathrm{D}$ ) cryoelectrolysis with cryoelectrolysis probe as the anode, $400 \mathrm{~mA} \mathrm{pH}$ front and ice front at a later time from panel $C, E)$ cryoelectrolysis with cryoelectrolysis probe as the cathode, $50 \mathrm{~mA}, \mathrm{~F}$ ) cryoelectrolysis with cryoelectrolysis probe as the cathode, $50 \mathrm{~mA}, \mathrm{pH}$ front and ice front at a later time from panel E,. Top photo earlier time. Bottom photo later time Black arrow - $\mathrm{pH}$ front, black dashed arrow - ice front, white line - interesting feature near the cryoelectrolysis probe. Photographs $A$ and $B, C$ and $D, E$ and $F$, are to the same scale. 


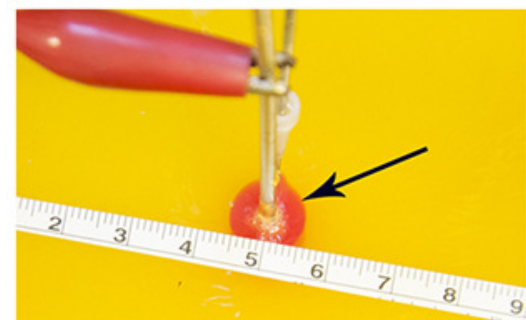

A
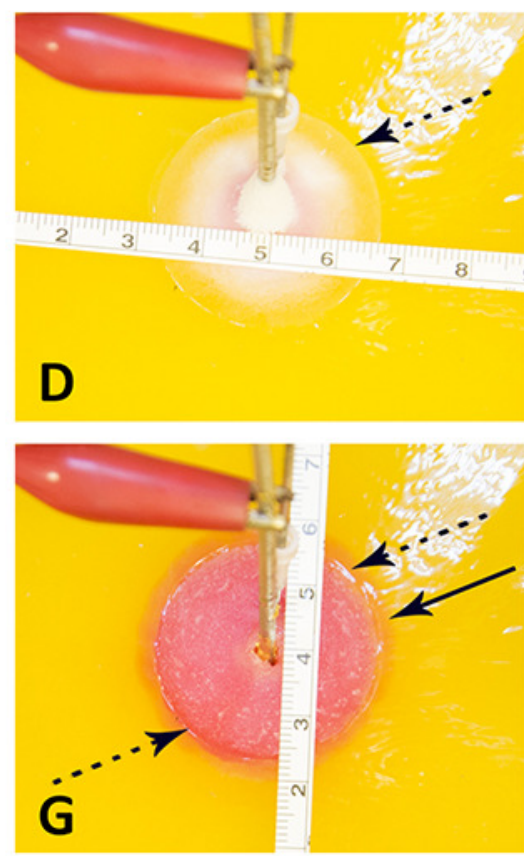
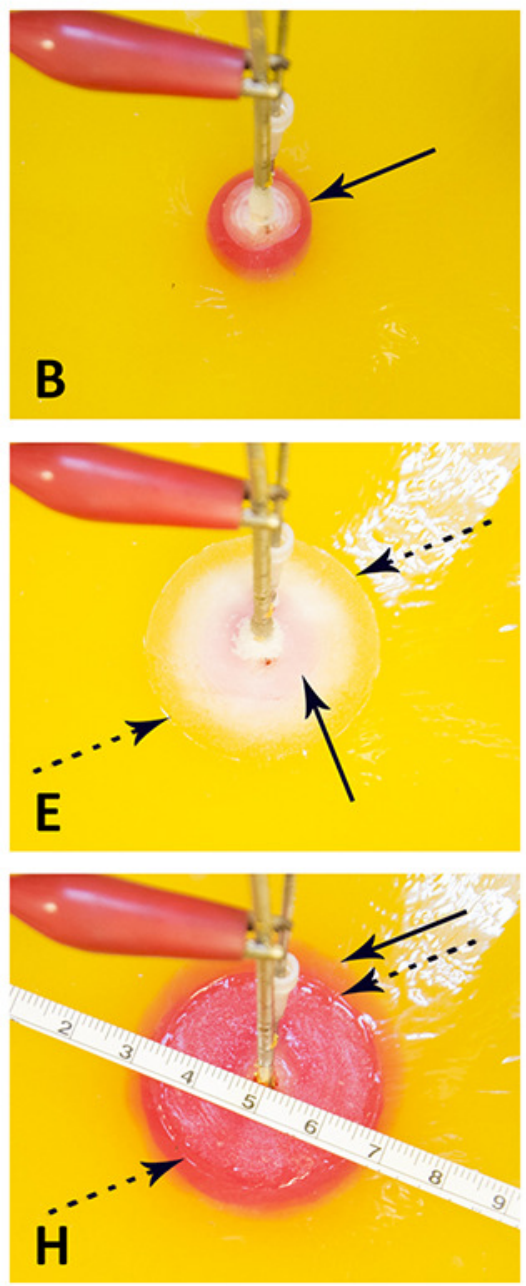
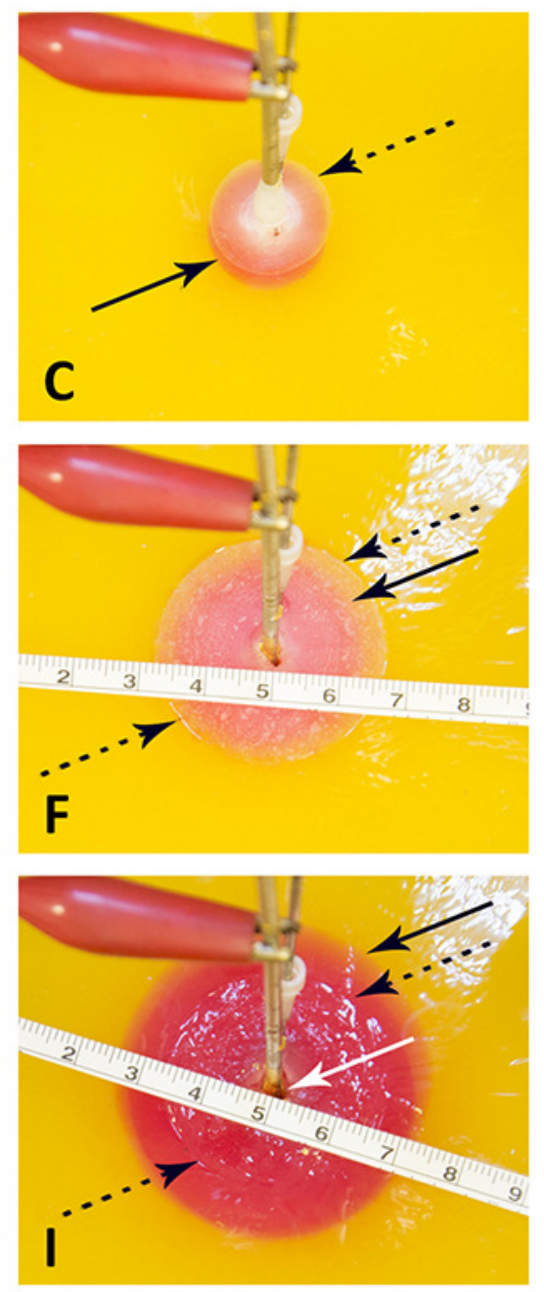

Figure 4: Progression of a $\mathrm{pH}$ front and a ice front during a typical cryoelectrolysis protocol. Results shown as a function of time after the start of the experiment (in minutes); A) $1 \mathrm{~min}, \mathrm{~B}) 2$ $\min$, C) $3.5 \mathrm{~min}$, D) $11 \mathrm{~min}$, E) $12.5 \mathrm{~min}$, F) $16 \mathrm{~min}, \mathrm{G}) 18.5 \mathrm{~min}, \mathrm{H})$ 21, I) $26 \mathrm{~min}$. All the figures are at the same scale (cm scale shown). The margin of the $\mathrm{pH}$ front is marked with a dark arrow and of the ice front with a dotted dark arrow. A feature of interest near the cryoelectrolysis probe marked with a white arrow. 


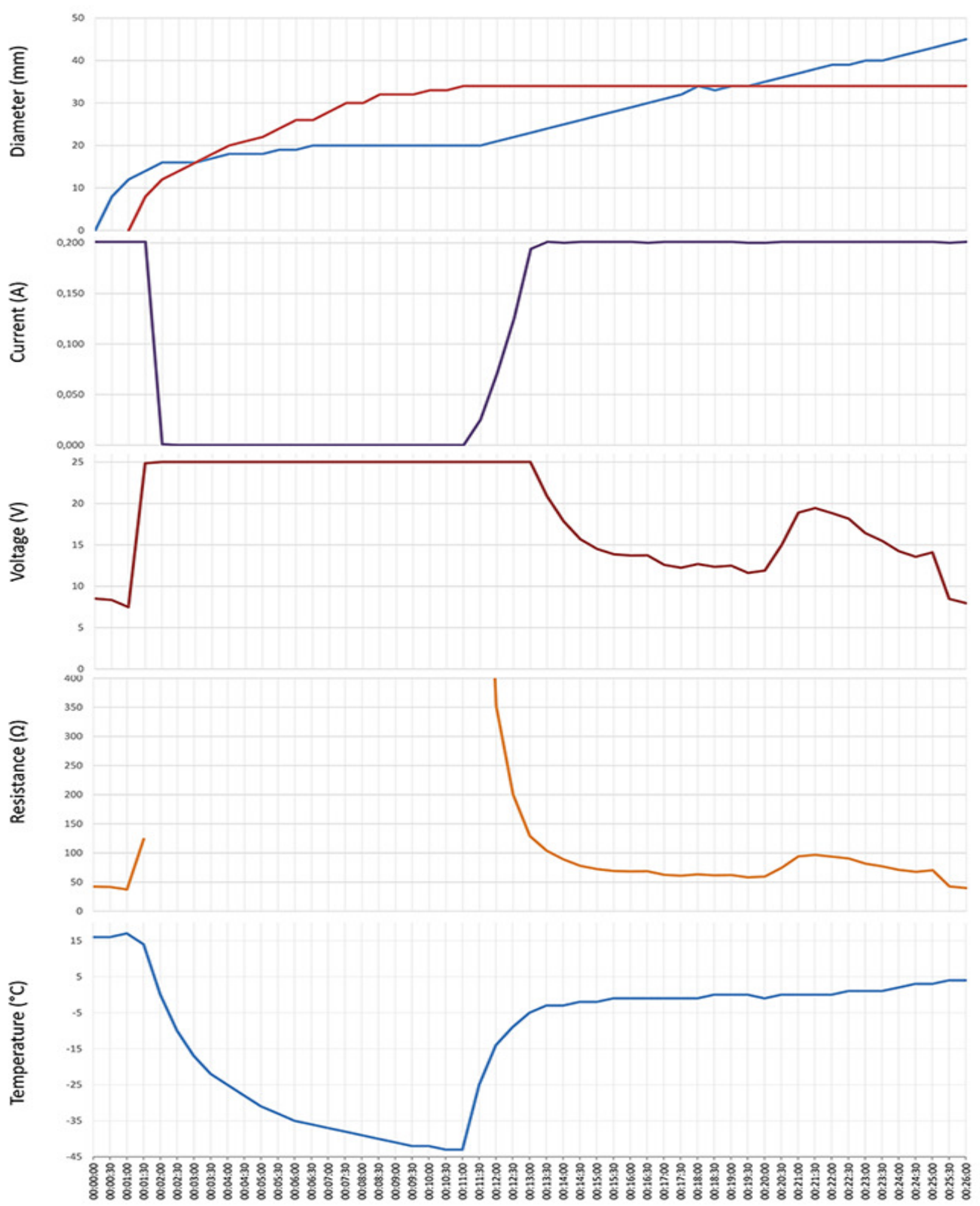

Figure 5: Data from an experiment in which the cryoelectrolysis probe served as the anode and 561 the preset current was $200 \mathrm{~mA}$. From top to bottom: the diameter of the ice front (green line) 562 and of the $\mathrm{pH}$ front (blue line); current; voltage; overall resistance; temperature, as a function of 563 time in minutes. 


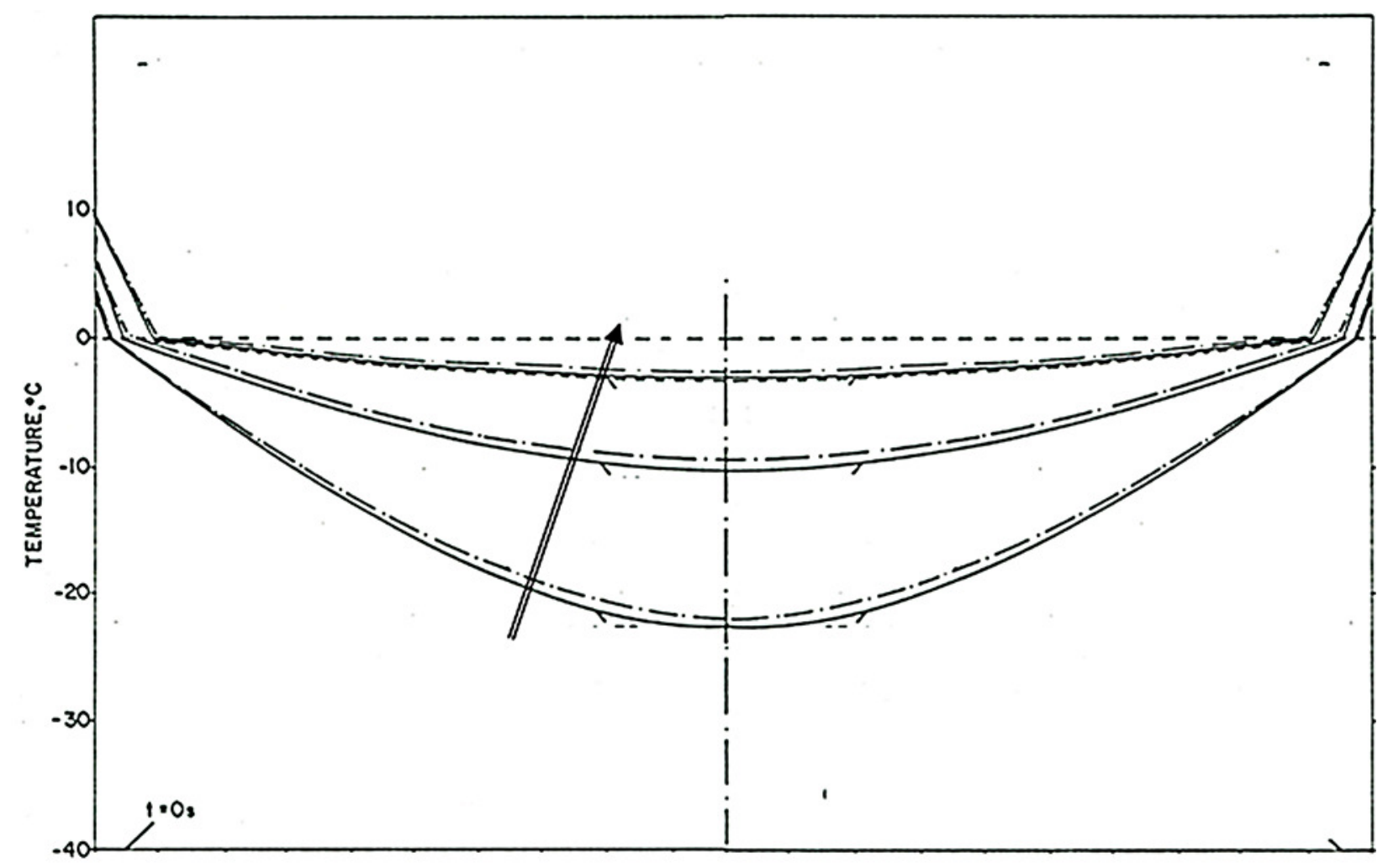

Figure 6: Qualitative depiction of the temperature distribution at various times during the thawing of a frozen cylinder of pure water, at an initial temperature of $-40{ }^{\circ} \mathrm{C}$, when the outer surface of the cylinder is $10 \mathrm{C}$. the time of the curves, increases in the direction of the arrow. The location of the interface between the frozen tissue and the unfrozen tissue domain corresponds to the $0{ }^{\circ} \mathrm{C}$ isotherm. The domain at a temperature lower than $0{ }^{\circ} \mathrm{C}$ is frozen. The figure is a 


\section{References}

574

575

576

577

Baust, J. G., W. Hollister, A. Mathews and R. Van Buskirk (1997). "Gene-regulated cell death follows cryosurgery." Cryobiology 35(4): 322-322.

578 Clarke, D. M., J. M. Baust, R. G. Van Buskirk and J. G. Baust (2001). "Chemo-cryo combination 579 therapy: An adjunctive model for the treatment of prostate cancer." Cryobiology 42(4): 274-

580285.

581 Fosh, B. G., J. G. Finch, A. A. Anthony, M. M. Lea, S. K. Wong, C. L. Black and G. J. Maddern

582 (2003). "Use of electrolysis for the treatment of non-resectable hepatocellular carcinoma." Anz 583 Journal of Surgery 73(12): 1068-1070.

584 Fosh, B. G., J. G. Finch, M. Lea, C. Black, S. Wong, S. Wemyss-Holden and G. J. Maddern (2002).

585 "Use of electrolysis as an adjunct to liver resection." British Journal of Surgery 89(8): 999-1002.

586 Gazelle, G. S., S. N. Goldberg, L. Solbiati and T. Livraghi (2000). "Tumor ablation with radio-

587 frequency energy." Radiology 217(3): 633-646.

588 Gilbert, J. C., G. M. Onik, W. K. Hoddick and B. Rubinsky (1984). "The use of ultrasound imaging 589 for monitoring cryosurgery." IEEE Transactions on Biomedical Engineering 31(8): 565-565.

590 Hong, J. S. and B. Rubinsky (1995). "Phase transformation in materials with non-uniform phase 591 transition temperatures. ." Journal of Heat Transfer-Transactions of the ASME 117(3): 803-805.

592 Ishiguro, H. and B. Rubinsky (1994). "Mechanical Interaction between ice crystals and red blood 593 cells during directional solidification." Cryobiology 31(5): 483-500.

594 Kennedy, L. C., L. R. Bickford, N. A. Lewinski, A. J. Coughlin, Y. Hu, E. S. Day, J. L. West and R. A. 595 Drezek (2011). "A New Era for Cancer Treatment: Gold-Nanoparticle-Mediated Thermal 596 Therapies." Small 7(2): 169-183.

597 Koushafar, H., L. Pham, C. Lee and B. Rubinsky (1997). "Chemical adjuvant cryosurgery with 598 antifreeze proteins." Journal of Surgical Oncology 66(2): 114-121.

599 Lugnani, F., F. Zanconati, T. Marcuzzo, C. Bottin, P. Mikus, E. Guenther, N. Klein, L. Rubinsky, M. 600 K. Stehling and B. Rubinsky (2015). "A Vivens Ex Vivo Study on the Synergistic Effect of 601 Electrolysis and Freezing on the Cell Nucleus." Plos One 10(12).

602 Mir, L. M. and B. Rubinsky (2002). "Treatment of cancer with cryochemotherapy." British 603 Journal of Cancer 86(10): 1658-1660.

604 Nilsson, E., H. von Euler, J. Berendson, A. Thorne, P. Wersall, I. Naslund, A. S. Lagerstedt, K. 605 Narfstrom and J. M. Olsson (2000). "Electrochemical treatment of tumours."

606 Bioelectrochemistry 51(1): 1-11.

607 Onik, G., C. Cooper, H. I. Goldberg, A. A. Moss, B. Rubinsky and M. Christianson (1984).

608 "Ultrasonic characteristics of frozen liver." Cryobiology 21(3): 321-328.

609 Phillips, M., N. Raju, L. Rubinsky and B. Rubinsky (2015). "Modulating electrolytic tissue ablation

610 with reversible electroporation pulses." Technology 3(1): 45-53.

611 Phillips, M., L. Rubinsky, A. Meir, N. Raju and B. Rubinsky (2015). "Combining Electrolysis and

612 Electroporation for Tissue Ablation." Technology in Cancer Research \& Treatment 14(4): 395-

613410.

614 Preciado, J. A., P. Shandakumaran, S. Cohen and B. Rubinsky (2003). Utilization of directional

615 freezing for the construction of tissue engineering scaffolds. ASME 2003 International

616 Mechanical Engineering Congress and Exposition, Washington DC. 
617 Rubinsky, B. (1983). "Solidification processes in saline solutions. ." Journal of Crystal Growth

618 62(3): 513-522.

619 Rubinsky, B. (2000). "Cryosurgery." Annual Review of Biomedical Engineering 2: 157-187.

620 Rubinsky, B., Ed. (2010). Irreversible electroporation. Series in Biomedical Engineering, Springer

621 Verlag.

622 Rubinsky, B. and E. G. Cravalho (1979). "Analysis for the temperature Distribution During the

623 Thawing of a Frozen Biological Organ." A.I.Ch.E. Symposium Series, 75: 81-88.

624 Rubinsky, B., J. C. Gilbert, G. M. Onik, M. S. Roos, S. T. S. Wong and K. M. Brennan (1993).

625 "Monitoring cryosurgery in the brain and in the protstate with proton NMR. ." Cryobiology

626 30(2): 191-199.

627 Rubinsky, B. and M. Ikeda (1985). "A cryosmicroscope using directional solidification for the

628 controlled freezing of biological matter. ." Cryobiology 22(1): 55-68.

629 Rubinsky, B., C. Y. Lee, J. Bastacky and T. L. Hayes (1987). "The mechanism of freezing in

630 biological tissue - the liver. ." Cryo-Letters 8(6): 370-381.

631 Rubinsky, B., C. Y. Lee, J. Bastacky and G. Onik (1990). "The process of freezing and the

632 mechanims of damage during hepatic cryosurgery. ." Cryobiology 27(1): 85-97.

633 Rubinsky, B. and D. E. Pegg (1988). "A mathematical model for the freezing process in biological

634 tissue. ." Proceedings of the Royal Society of London Series B-Biological Sciences 234(1276):

635 343-349.

636 Rubinsky, L., E. Guenther, P. Mikus, M. Stehling, K., and B. Rubinsky (2015). "Electrolytic Effect

637 During Tissue Ablation by Electroporation." Technology in cancer Research and Treatement.

638 Rubinsky, L., E. Guenther, P. Mikus, M. Stehling and B. Rubinsky (2016). "Electrolytic Effects

639 During Tissue Ablation by Electroporation." Technology in Cancer Research \& Treatment 15(5):

640 NP95-NP103.

641 Stehling, M. K., E. Guenther, P. Mikus, N. Klein, L. Rubinsky and B. Rubinsky (2016). "Synergistic

642 Combination of Electrolysis and Electroporation for Tissue Ablation." Plos One 11(2).

643 